\title{
A method of measuring parameters of an extensive air shower at Yakutsk EAS array
}

\section{Lev Timofeev* SHICRA SB RAS}

E-mail: timofeevleveikfia.sbras.ru

\begin{abstract}
Proposed a new method for measuring the cherenkov light from the extensive air shower (EAS) of cosmic rays (CR), which allows to determine not only the primary particle energy and angle of arrival, but also the parameters of the shower in the atmosphere - the maximum depth and "age". For measurements Cherenkov light produced by EAS is proposed to use a ground network of cherenkov telescopes which are mounted on drones with the regulated distance depending on the total number of telescopes operating in the coincidence signals and current task, acting autonomously, or includes a detector of the charged components, radio waves, etc. as part of EAS array. In a results such array could developed, energy measurement and CR. This is particularly important in the study of galactic cosmic ray in $\mathrm{E}>10^{14} \mathrm{eV}$, where currently there are no direct measurements of the maximum depth of the EAS.
\end{abstract}

38th International Conference on High Energy Physics 3-10 August 2016

Chicago, USA

${ }^{*}$ Speaker. 


\section{Introduction}

The only way to study the properties of cosmic rays with energy above $10^{15} \mathrm{eV}$ is the registration of Extensive Air Shower generated by such primary particles, in various ways. The most developed and effective technique is to use EAS array. The advantage of ground array is a wide range of detected energy cosmic rays, the ability to detect different types of shower of secondary particles (shower components) as well as their relative cheapness compared with alternative methods (space observation). EAS cherenkov radiation is one of the main sources of information about the properties of ultrahigh energy cosmic rays. Investigation of Cherenkov light induced by cosmic rays in the atmosphere began in the middle of the previous century in the UK and USSR. An exhaustive description of early developments is given in the book [1], while recent reviews of the whole area are given, e.g., in Refs. [2,3]. Ground EAS array require a different kind of communication, and thus imply the existence of the village. One of the most common problems during the registration of optical methods is stray light photomultiplier tubes that act as detectors. Highlights reduces uptime and aperture detectors or significantly increases the detection threshold, makes it difficult to calibrate the instrument, so - reduces Statistics. A possible way out of the situation could be the installation of completely or partially consisting of cherenkov detectors installed on unmanned aerial vehicles (eg quadrocopters). If necessary, the location of such units may be changed quickly without additional financial cost, which would increase the useful time of registration. In addition, the configuration of these type arrays can be easily adjusted in accordance with the scientific problems (eg, change in the energy range of cosmic rays detected). Also, being in the harsh conditions of the field will be reduced to the minimum necessary (to direct observation time), which will reduce wear and tear and accidental incidents.

\section{Project goals}

The project aims to improve the effectiveness of the experimental equipment used in solving problems of fundamental research. This is achieved by the fact that the cherenkov light detectors are mounted on drones that will variously configure the system. At now there is no usage of drones to detecting Cherenkov light of Extensive Air Showers in the world. Since detector will be not linked to the location, these allow us to change the configuration of the existing EAS, increase the density of the detectors or the array area in any form due to perform specific tasks. Particularly relevant mobile detectors in a neighborhood of human settlements, creating a parasitic light background, and as a consequence reduce operation time for observation and statistics. Increased, the distance between detectors and settlement, we will increase the time of observation and statistics, respectively. During registration, an unmanned aerial vehicle will be on the ground in order to reduce battery consumption and noise generated by operation of the motor. Immediately prior to the surveillance drones mounted with detectors will move and landed on observing these geotochki, and after the end of the observation back to the central register for recharging. Reducing the time spent in harsh field conditions to the necessary minimum which significantly reduce the wear of instruments, as well as protect against accidental incidents (natural disasters, accidents). Data could be transmitted on WiMax and GSM standards, to obtain information in real time, the stored information also can be read out manually during daytime charging. GPS units will also be used for 
time synchronization. It is supposed to use passive power supplies at low temperatures would be wise to use $\mathrm{LiFePO}_{4}$ batteries have less capacity, but resistant to low temperatures. As an observing camera for the first time at the Yakutsk EAS will be tested solid silicon photomultipliers see Ref [4], have low weight and low power consumption (12 V low voltage compared with conventional photomultiplier tubes $1-2 \mathrm{kV}$ ), for which the price is high but constantly decreasing. Thus, we not only improve the potential of extensive air showers array, but also lay the foundation for future upgrades and possibly for a new type of arrays, consisting of the detectors installed on drones, which can be applied anywhere. Possibility of bidirectional communication at a distance lets you organize events filtration system, which will save space on the storage media.

\section{Conclusion}

One of the unsolved problems of the origin of ultrahigh-energy cosmic rays is a massive part of the nuclei in cosmic rays. There are conflicting data on the average weight of the particles obtained by measuring the maximum depth of the EAS and its fluctuations according to the Pierre Auger Observatory, Telescope Array and the Yakutsk EAS array Ref [5]. Measurement of Cherenkov light detectors on the mobile platform will be held for the first time in the world. Experimental study of cosmic rays with such energies is possible only through registration of the EAS - a cascade of secondary particle generated particles of the primary cosmic radiation as a result of the strong and electroweak interactions with atomic nuclei in the atmosphere, since the intensity of the primary cosmic radiation is extremely low. Above all, remain unidentified composition and cosmic ray sources. These experiments HiRes (USA) and the Telescope Array indicate the proton composition of cosmic rays with energy above $10^{19} \mathrm{eV}$. Then the data of the Yakutsk EAS, the share of the muon, and the Pierre Auger Observatory at maximum depth, indicate an increase in the proportion of heavy nuclei with increasing energy at these energies. Despite the relatively small Statistics (several thousand events), this contradiction is quite significant.

\section{Acknowledgments}

The work is supported in part by RFBR (grants 11-02-00158, 11-02-12193, 12-02-10005, 1202-31550) and the Russian Ministry of Education and Science (contracts 02.740.11.0248, 16.518.11.7075). Grant "Scientific and Educational Foundation for Young Scientist of Republic of Sakha(Yakutia)" 201604010203 and Head of Sakha Republic Grant for young scientist 17/16.

\section{References}

[1] J.V. Jelley, Cherenkov Radiation and its Applications (Pergamon Press, 1958)

[2] A.S. Lidvansky, Radiation Physics and Chemistry 7589 (2006)

[3] A.A. Watson, astro-ph/1101.4535.

[4] http://www.ait-instruments.com

[5] E. Barcikowski et al., Mass composition working group report. EPJ Web of Conferences 53, 2013 01006 